\title{
MULTIPLICITY OF POSITIVE SOLUTIONS TO SEMILINEAR ELLIPTIC BOUNDARY VALUE PROBLEMS
}

\author{
KENICHIRO UMEZU
}

Received 12 September 1999

We study semilinear elliptic boundary value problems of one parameter dependence where the number of positive solutions is discussed. Our main purpose is to characterize the critical value given by the infimum of such parameters for which positive solutions exist. Our approach is based on super- and sub-solutions, and relies on the topological degree theory on the positive cones of ordered Banach spaces. A concrete example is also presented.

\section{Introduction}

Let $D$ be a bounded domain of Euclidean space $\mathbb{R}^{N}, N \geq 2$, with smooth boundary $\partial D$. In this paper, we study the following semilinear elliptic boundary value problem:

$$
\begin{gathered}
L u:=(-\Delta+c(x)) u=\lambda f(u) \quad \text { in } D, \\
B u:=a(x) \frac{\partial u}{\partial \mathbf{n}}+(1-a(x)) u=0 \quad \text { on } \partial D .
\end{gathered}
$$

Here

(1) $\Delta$ denotes the usual Laplacian $\sum_{j=1}^{N} \partial^{2} / \partial x_{j}^{2}$ in $\mathbb{R}^{N}$,

(2) $c \in C^{\infty}(\bar{D})$ and $c>0$ in $D$,

(3) $\lambda$ is a positive parameter,

(4) $f$ is a real-valued, nonnegative $C^{1}$-function on $[0, \infty)$,

(5) $B$ is a degenerate boundary operator with coefficient $a \in C^{\infty}(\partial D)$ satisfying

$$
0 \leq a(x) \leq 1 \quad \text { on } \partial D \text {, }
$$

(6) $\mathbf{n}$ is the exterior unit normal to $\partial D$.

The degeneracy means that the so-called Shapiro-Lopatinskii condition breaks down at $x \in \partial D$ where $a(x)=0$ if $a \neq \equiv 0$ on $\partial D$. We note that our boundary condition is the Dirichlet one if $a \equiv 0$ on $\partial D$ and Neumann one if $a \equiv 1$ on $\partial D$.

Copyright (c) 1999 Hindawi Publishing Corporation Abstract and Applied Analysis 4:3 (1999) 195-208 1991 Mathematics Subject Classification: 35J65 URL: http://aaa.hindawi.com/volume-4/S1085337599000147.html 
196 Semilinear elliptic boundary value problems

A function $u \in C^{2}(\bar{D})$ is called a solution of (1.1) if it satisfies (1.1). A solution of (1.1) which is positive everywhere in $D$ is called positive.

In this paper, we consider the existence and multiplicity of positive solutions of (1.1).

Here we assume for nonlinear $f$ that

$$
\begin{aligned}
& f(0)=0, \\
& f^{\prime}(0)=1 .
\end{aligned}
$$

In addition to (1.3) and (1.4), if the condition

$$
f(t) \leq t, \quad \forall t \geq 0,
$$

is assumed, then Green's formula gives us a necessary condition for the existence of a positive solution as follows:

$$
\lambda \geq \lambda_{1}
$$

Here $\lambda_{1}$ is the first eigenvalue of the eigenvalue problem

$$
\begin{aligned}
& L \varphi=\lambda \varphi \quad \text { in } D, \\
& B \varphi=0 \quad \text { on } \partial D .
\end{aligned}
$$

It is known (see [4]) that $\lambda_{1}$ is positive and simple, and that the corresponding eigenfunction can be chosen to be positive in $D$. We denote by $\varphi_{1}$ the positive eigenfunction normalized as $\left\|\varphi_{1}\right\|_{\infty}=1$, where $\|\cdot\|_{\infty}$ is the maximum norm of space $C(\bar{D})$ of continuous functions over $\bar{D}$.

In addition to (1.3), (1.4), and (1.5), if the concavity is given for $f$, more precisely, if $f(t) / t$ is strictly decreasing with respect to $t>0$, then the super-sub-solution method leads to the assertion that if $\lambda \in\left(\lambda_{1}, \lambda_{1} / \alpha\right)$ where $\alpha=\lim _{t \rightarrow \infty} f(t) / t$, then problem (1.1) has a unique positive solution, and otherwise, there is no positive solution of (1.1) (see [7, Corollary 2]).

This paper is mainly concerned with the case where $f$ is convex with respect to $t>0$ small and sublinear, that is, there exists a constant $0<t_{0}<1$ with the conditions

$$
\begin{aligned}
& f(t)>t, \quad \forall t \in\left(0, t_{0}\right], \\
& f(t) \leq t, \quad \forall t \in\left[1 / t_{0}, \infty\right) .
\end{aligned}
$$

If $f$ satisfies (1.3), (1.4), (1.8), and (1.9), then we denote by $\bar{f}, f_{\infty}$ the constants given respectively by

$$
\begin{gathered}
\bar{f}=\sup _{t>0} \frac{f(t)}{t}, \\
f_{\infty}=\limsup _{t \rightarrow \infty} \frac{f(t)}{t} .
\end{gathered}
$$

Now, we can formulate our main results. The first one is the following existence and multiplicity theorem for positive solutions of (1.1). 
THEOREM 1.1. Let conditions (1.3), (1.4), (1.8), and (1.9) be satisfied. Then there exists a constant $\Lambda \in\left[\lambda_{1} / \bar{f}, \lambda_{1}\right)$ such that problem (1.1) has at least one positive solution for every $\lambda \in\left[\Lambda, \lambda_{1} / f_{\infty}\right)$ and no positive solution for any $\lambda \in(0, \Lambda)$ and, moreover, there exist at least two positive solutions of (1.1) for each $\lambda \in\left(\Lambda, \lambda_{1}\right)$.

Remark 1.2. By (1.8) we note

$$
\bar{f}>1
$$

and we find from (1.9) and the condition that $f$ is nonnegative, that

$$
0 \leq f_{\infty} \leq 1
$$

If $f_{\infty}=0$, then it is understood in Theorem 1.1 that $\lambda_{1} / f_{\infty}=\infty$.

If we restrict our consideration to the nondegenerate case where either $a \equiv 0$ or $0<a \leq 1$, then Lions [3, Theorem 1.4] studied the case $f_{\infty}=0$, where a topological degree argument is employed. We also refer to Ambrosetti, Brézis, and Cerami [2] for a class of $f$ which has concavity for small values $t>0$ and convexity for large values $t>0$, where the variational method is used as well as the super-sub-solution method.

However, our main interest here is to characterize the critical value $\Lambda$. Let $e \in$ $C^{\infty}(\bar{D})$ be a unique solution of the problem

$$
\begin{array}{cc}
L u=1 & \text { in } D, \\
B u=0 & \text { on } \partial D .
\end{array}
$$

It is known [5, Lemma 2.1] that the solution $e$ satisfies

$$
\begin{aligned}
& e>0 \quad \text { in } \bar{D} \backslash \Gamma_{0}, \\
& \frac{\partial e}{\partial \mathbf{n}}<0 \quad \text { on } \Gamma_{0},
\end{aligned}
$$

where $\Gamma_{0}=\{x \in \partial D: a(x)=0\}$.

Now the second main result of ours is the following.

TheOREM 1.3. Let $\beta$ be the positive constant defined by (3.7). In addition to (1.3), (1.4), (1.8), and (1.9), we suppose that $f$ is nondecreasing with respect to $t>0$. If $f$ satisfies the condition

$$
\bar{f}>\frac{\|e\|_{\infty}}{\beta},
$$

then problem (1.1) has at least two positive solutions for every

$$
\frac{1}{\bar{f} \beta}<\lambda<\frac{1}{\|e\|_{\infty}},
$$

so that the critical value $\Lambda$ given by Theorem 1.1 has the following estimate:

$$
\frac{\lambda_{1}}{\bar{f}} \leq \Lambda \leq \frac{1}{\bar{f} \beta} .
$$


198 Semilinear elliptic boundary value problems

Remark 1.4. The maximum principle ensures that $1 /\|e\|_{\infty} \leq \lambda_{1} \leq 1 / \beta$ (cf. $[9$, Lemma 4.2]). Moreover, we can show (see [10, Corollary 5.3]) that, under the Neumann condition $a \equiv 1$, we have

$$
\lambda_{1}=\frac{1}{\beta} .
$$

Estimate (1.18) would be therefore optimal in this sense.

The rest of this paper is organized as follows: Section 2 is devoted to the proof of Theorem 1.1. Our main tool for the discussion of the multiplicity of positive solutions is the three fixed point existence theorem for compact, strongly increasing mappings in ordered Banach spaces due to Amann [1, Theorem 14.2]. Section 3 contains the proof of Theorem 1.3. For this we use Wiebers' result [9, Lemma 4.4], based on the topological degree theory on the positive cones of ordered Banach spaces. In Section 4 we give an example of $f$ satisfying the assumption of Theorem 1.3 and discuss the existence and multiplicity of positive solutions.

\section{Proof of Theorem 1.1}

This section is devoted to the proof of Theorem 1.1. First we reduce (1.1) to the equation of a compact, strongly increasing mapping in the positive cone of an ordered Banach space. For this we begin by recalling the following two existence and uniqueness theorems for the linear degenerate boundary value problem

$$
\begin{gathered}
L u=h \quad \text { in } D, \\
B u=0 \quad \text { on } \partial D .
\end{gathered}
$$

Theorem 2.1 (see [6, Theorem 1.1]). The mapping

$$
\begin{aligned}
(L, B): C^{2+\theta}(\bar{D}) & \longrightarrow C^{\theta}(\bar{D}) \times C_{*}^{1+\theta}(\partial D), \\
u & \longmapsto(L u, B u)
\end{aligned}
$$

is an algebraic and topological isomorphism for $0<\theta<1$. Here $C^{m+\theta}(\bar{D})$ denotes the usual Hölder space with norm $\|\cdot\|_{C^{m+\theta}(\bar{D})}$ if $m$ is a nonnegative integer, and $C_{*}^{1+\theta}(\partial D)$ is an interpolation space associated with the boundary operator $B$ in the following sense:

$$
C_{*}^{1+\theta}(\partial D)=\left\{\varphi=a \varphi_{1}+(1-a) \varphi_{0}: \varphi_{i} \in C^{2-i+\theta}(\partial D)\right\} .
$$

We can verify that $C_{*}^{1+\theta}(\partial D)$ is a Banach space with the norm

$$
\begin{aligned}
\|\varphi\|_{C_{*}^{1+\theta}(\partial D)}=\inf \left\{\left\|\varphi_{1}\right\|_{C^{1+\theta}(\partial D)}+\left\|\varphi_{0}\right\|_{C^{2+\theta}(\partial D)}\right. \\
\left.: \varphi=a \varphi_{1}+(1-a) \varphi_{0}, \varphi_{i} \in C^{2-i+\theta}(\partial D)\right\} .
\end{aligned}
$$

Theorem 2.2 (see [8, Theorem 1]). The mapping

$$
\begin{aligned}
(L, B): W^{2, p}(D) & \longrightarrow L^{p}(D) \times W_{*}^{1-(1 / p), p}(\partial D), \\
u & \longmapsto(L u, B u)
\end{aligned}
$$


is an algebraic and topological isomorphism for $1<p<\infty$. Here $W^{m, p}(D)$ denotes the usual Sobolev space with norm $\|\cdot\|_{W^{m, p}(D)}$ if $m$ is a nonnegative integer, $L^{p}(D)=$ $W^{0, p}(D)$, and $W_{*}^{1-(1 / p), p}(\partial D)$ is an interpolation space given by

$$
W_{*}^{1-(1 / p), p}(\partial D)=\left\{\varphi=a \varphi_{1}+(1-a) \varphi_{0}: \varphi_{i} \in W^{2-i-(1 / p), p}(\partial D)\right\},
$$

where $W^{2-i-(1 / p), p}(\partial D)$ is a Banach space given by

$$
W^{2-i-(1 / p), p}(\partial D)=\left\{\varphi=\left.u\right|_{\partial D}: u \in W^{2-i, p}(D)\right\}, \quad i=0,1,
$$

with the norm

$$
\|\varphi\|_{W^{2-i-(1 / p), p(\partial D)}}=\inf \left\{\|u\|_{W^{2-i, p}(D)}: u \in W^{2-i, p}(D),\left.u\right|_{\partial D}=\varphi\right\} .
$$

We can check that $W_{*}^{1-(1 / p), p}(\partial D)$ is a Banach space with the norm

$$
\begin{aligned}
\|\varphi\|_{W_{*}^{1-(1 / p)}(\partial D)}=\inf \left\{\left\|\varphi_{1}\right\|_{W^{1-(1 / p), p}(\partial D)}+\left\|\varphi_{0}\right\|_{W^{2-(1 / p), p}(\partial D)}\right. \\
\left.: \varphi=a \varphi_{1}+(1-a) \varphi_{0}, \varphi_{i} \in W^{2-i-(1 / p), p}(\partial D)\right\} .
\end{aligned}
$$

Let

$$
C_{B}^{2+\theta}(\bar{D})=\left\{u \in C^{2+\theta}(\bar{D}): B u=0 \text { on } \partial D\right\} .
$$

By Theorem 2.1, there exists the resolvent $K: C^{\theta}(\bar{D}) \rightarrow C_{B}^{2+\theta}(\bar{D})$ for (2.1), meaning that $K h$ is a unique solution of (2.1) for any $h \in C^{\theta}(\bar{D})$. By the well-known argument, Theorem 2.2 allows $K$ to be extended uniquely to the space $L^{p}(D), 1<p<\infty$. Especially, $K$ maps $C(\bar{D})$ compactly into $C^{1}(\bar{D})$ thanks to the Sobolev imbedding theorem. Furthermore, we can show (see [5, Lemma 2.1]) that $K$ is strictly positive, that is, $K h$ has property (1.15) for any $h \in P \backslash\{0\}$ where $P=\{u \in C(\bar{D}): u \geq 0$ on $\bar{D}\}$.

Let

$$
C_{e}(\bar{D})=\{u \in C(\bar{D}): \text { there exists a constant } c>0 \text { such that }-c e \leq u \leq c e \text { on } \bar{D}\},
$$

where $e$ is the unique positive solution of (1.14). It is easily seen that $C_{e}(\bar{D})$ is a Banach space with the norm

$$
\|u\|_{e}=\inf \{c>0:-c e \leq u \leq c e \text { on } \bar{D}\} .
$$

Letting

$$
P_{e}=P \cap C_{e}(\bar{D}),
$$

we see that $P_{e}$ has nonempty interior. We can, moreover, show (see [5, Proposition 2.2]) that $K$ is strongly positive, that is, $K h$ is an interior point of $P_{e}$, denoted by $K h \in \stackrel{\circ}{P}_{e}$, for any $h \in P \backslash\{0\}$.

The standard regularity argument due to Theorems 2.1 and 2.2 shows that problem (1.1) is equivalent to the equation

$$
u=F(\lambda, u):=\lambda K f(u) \quad \text { in } C(\bar{D}) .
$$


Here we see that $F:(0, \infty) \times P \rightarrow P$ is compact, since $K$ is compact and strictly positive, and since $f$ is nonnegative. Since $f \in C^{1}([0, \infty))$, for any $t_{1}>0$ there exists a constant $k>0$ such that $f(t)+k t$ is strictly increasing in $t \in\left[0, t_{1}\right]$. This shows that

$$
F_{k}(\lambda, u):=\lambda K_{k}(f(u)+k u), \quad(\lambda, u) \in(0, \infty) \times P
$$

is strongly increasing in $u \in P_{t_{1}}$ where

$$
P_{t}=\{u \in P: u \leq t \text { on } \bar{D}\}, \quad t>0
$$

which means that $F_{k}(\lambda, u)-F_{k}(\lambda, v) \in \stackrel{\circ}{P}_{e}$ for any $u, v \in P_{t_{1}}$ satisfying $u-v \in P \backslash\{0\}$. Here $K_{k}$ is the resolvent for the problem

$$
\begin{gathered}
(L+k) u=h \text { in } D, \\
B u=0 \text { on } \partial D .
\end{gathered}
$$

Summing up, we see that problem (1.1) is equivalent to the equation

$$
u=F_{k}(\lambda, u) \quad \text { in } C(\bar{D})
$$

and also we can verify that problem (1.1) is equivalent to the equation

$$
u=F_{k}(\lambda, u) \text { in } C_{e}(\bar{D})
$$

We remark here that the condition that $F_{k}$ is strongly increasing in $C_{e}(\bar{D})$ plays a crucial role in the discussion of the multiplicity of positive solutions of (1.1).

Now we prove Theorem 1.1. By use of the local bifurcation theory from simple eigenvalues in the degenerate case [4], condition (1.8) shows that there exists a positive solution of (1.1) for every $\lambda \in\left(\lambda_{1}-\delta, \lambda_{1}\right)$ with some $\delta>0$ small. So, let $\Lambda$ be the positive constant defined as

$$
\Lambda=\inf \left\{\lambda<\lambda_{1}:(1.1) \text { has at least one positive solution }\right\} \text {. }
$$

Here we assert that

$$
\Lambda \geq \frac{\lambda_{1}}{\bar{f}}
$$

where $\bar{f}$ is given by (1.10). Indeed, Green's formula shows

$$
\int_{D}\left(L u \cdot \varphi_{1}-u \cdot L \varphi_{1}\right) d x=\int_{\partial D}\left(\frac{\partial \varphi_{1}}{\partial \mathbf{n}} u-\frac{\partial u}{\partial \mathbf{n}} \varphi_{1}\right) d \sigma
$$

for any positive solution $u$ of (1.1). Here $d \sigma$ is the surface element of $\partial D$. From the boundary conditions

$$
\begin{array}{cc}
a \frac{\partial u}{\partial \mathbf{n}}+(1-a) u=0 & \text { on } \partial D, \\
a \frac{\partial \varphi_{1}}{\partial \mathbf{n}}+(1-a) \varphi_{1}=0 & \text { on } \partial D
\end{array}
$$


we note that

$$
\left(\begin{array}{cc}
\frac{\partial u}{\partial \mathbf{n}} & u \\
\frac{\partial \varphi_{1}}{\partial \mathbf{n}} & \varphi_{1}
\end{array}\right)\left(\begin{array}{c}
a \\
1-a
\end{array}\right)=\left(\begin{array}{l}
0 \\
0
\end{array}\right) \quad \text { on } \partial D .
$$

Since $(a, 1-a) \neq(0,0)$ on $\partial D$, we necessarily obtain

$$
\frac{\partial \varphi_{1}}{\partial \mathbf{n}} u-\frac{\partial u}{\partial \mathbf{n}} \varphi_{1}=0 \quad \text { on } \partial D
$$

Consequently,

$$
\int_{D}\left(L u \cdot \varphi_{1}-u \cdot L \varphi_{1}\right) d x=0
$$

Meanwhile, we obtain

$$
0=\int_{D}\left(L u \cdot \varphi_{1}-u \cdot L \varphi_{1}\right) d x \leq\left(\lambda \bar{f}-\lambda_{1}\right) \int_{D} u \varphi_{1} d x
$$

which implies assertion (2.21).

To show the existence of a positive solution of (1.1) for $\lambda \in\left(\Lambda, \lambda_{1} / f_{\infty}\right)$, we use the super-sub-solution method. However we consider only the case $f_{\infty}>0$. The case $f_{\infty}=0$ can be verified in the same manner with a minor modification. A nonnegative function $\psi \in C^{2}(\bar{D})$ is said to be a super-solution of (1.1) if we have

$$
\begin{gathered}
L \psi \geq \lambda f(\psi) \quad \text { in } D, \\
B \psi \geq 0 \text { on } \partial D .
\end{gathered}
$$

A nonnegative function $\phi \in C^{2}(\bar{D})$ is said to be a sub-solution of (1.1) if we have

$$
\begin{gathered}
L \phi \leq \lambda f(\phi) \quad \text { in } D \\
B \phi \leq 0 \quad \text { on } \partial D
\end{gathered}
$$

A super-solution which is not a solution is called strict. Strict sub-solutions are defined similarly.

For any $\lambda \in\left(\Lambda, \lambda_{1} / f_{\infty}\right)$, there exists a constant $\varepsilon_{1}>0$ such that

$$
\lambda\left(f_{\infty}+\varepsilon_{1}\right)<\lambda_{1}
$$

and, from (1.11), we can choose a constant $d_{1}>0$ such that

$$
\lambda f(t)<\lambda\left(f_{\infty}+\varepsilon_{1}\right) t+d_{1}, \quad t \geq 0 .
$$

To construct super- and sub-solutions, we prove the following lemma.

Lemma 2.3. Let $\lambda \in\left(\Lambda, \lambda_{1} / f_{\infty}\right)$, and let $\varepsilon_{1}, d_{1}$ be the constants given by (2.30) and (2.31), respectively. Then the linear nonhomogeneous problem

$$
\begin{gathered}
L u=\lambda\left(f_{\infty}+\varepsilon_{1}\right) u+d_{1} \quad \text { in } D, \\
B u=0 \quad \text { on } \partial D
\end{gathered}
$$


has exactly one positive solution $\psi(\lambda) \in C^{2}(\bar{D})$. Furthermore, the positive solution $\psi(\lambda)$ is a strict super-solution of (1.1), satisfying

$$
u<\psi(\lambda) \text { in } D
$$

for any positive solution $u$ of (1.1) with parameter $\mu \in[\Lambda, \lambda)$.

Proof. Thanks to the positivity lemma [7, Lemma], condition (2.30) shows that problem (2.32) has exactly one positive solution. It follows from (2.31) that the positive solution $\psi(\lambda)$ is a strict super-solution of (1.1). For any positive solution $u$ of (1.1) with parameter $\mu \in[\Lambda, \lambda)$, we obtain

$$
\begin{gathered}
L(\psi(\lambda)-u)>\lambda\left(f_{\infty}+\varepsilon_{1}\right)(\psi(\lambda)-u) \text { in } D, \\
B(\psi(\lambda)-u)=0 \text { on } \partial D,
\end{gathered}
$$

where we have used (2.31) and the fact that $f$ is nonnegative. Using the positivity lemma again, we have (2.33) and the proof of Lemma 2.3 is complete.

From the definition of $\Lambda$ it follows that, for any $\lambda \in\left(\Lambda, \lambda_{1} / f_{\infty}\right)$, there exists a $\mu \in[\Lambda, \lambda)$ such that problem (1.1) with parameter $\mu$ has a positive solution $u_{\mu}$. Since $f$ is nonnegative, we see that $u_{\mu}$ is a sub-solution of (1.1). By (2.33) we obtain that $u_{\mu} \leq \psi(\lambda)$ on $\bar{D}$. The super-sub-solution method [6, Theorem 1] shows that problem (1.1) has at least one positive solution.

Next, we verify the existence of a positive solution of (1.1) for $\lambda=\Lambda$. By the definition of $\Lambda$, we can choose functions $u_{j} \in C^{2}(\bar{D})$ such that $u_{j}$ is a positive solution of (1.1) with parameter $\mu_{j}$ where $\mu_{j} \downarrow \Lambda$ as $j \rightarrow \infty$. It follows that $\left\|u_{j}\right\|_{\infty}$ is uniformly bounded. Indeed, we may assume

$$
\mu_{1}<\gamma:=\frac{\lambda_{1}+\Lambda}{2}
$$

and then, for the positive solution $\psi(\gamma)$ to (2.32) with $\lambda=\gamma$, we have $u_{j} \leq \psi(\gamma)$ on $\bar{D}$ for any $j \geq 1$, by virtue of (2.33).

By the regularity argument, $\left\|u_{j}\right\|_{C^{2+\theta}}$ is also uniformly bounded. Thanks to AscoliArzelà's theorem, we may assert, without loss of generality, that there is a function $\hat{u} \in C^{2}(\bar{D})$ such that

$$
u_{j} \longrightarrow \hat{u} \quad \text { in } C^{2}(\bar{D})
$$

which implies that

$$
\begin{gathered}
L \hat{u}=\Lambda f(\hat{u}) \quad \text { in } D, \\
\hat{u} \geq 0 \quad \text { in } D, \\
B \hat{u}=0 \quad \text { on } \partial D .
\end{gathered}
$$

It is known (see [1, Theorem 18.1]) that $\Lambda$ is an eigenvalue of (1.7) with a positive eigenfunction if $\Lambda$ is a bifurcation point from the line of the trivial solutions. Since $\Lambda<\lambda_{1}$, we obtain that $\hat{u} \not \equiv 0$. Hence the strong maximum principle shows

$$
\hat{u}>0 \text { in } D \text {. }
$$


Finally we consider the multiplicity of (1.1) for $\lambda \in\left(\Lambda, \lambda_{1}\right)$. We recall that for any $\lambda \in\left(\Lambda, \lambda_{1}\right)$ there exists a constant $\mu \in[\Lambda, \lambda)$ such that problem (1.1) with parameter $\mu$ admits a positive solution $u_{\mu}$. We see that $u_{\mu}$ is a strict sub-solution of (1.1). For positive constants $\varepsilon$, we have

$$
L\left(\varepsilon \varphi_{1}\right)-\lambda f\left(\varepsilon \varphi_{1}\right)=\left(\lambda_{1}-\lambda \frac{f\left(\varepsilon \varphi_{1}\right)}{\varepsilon \varphi_{1}}\right) \varepsilon \varphi_{1} \quad \text { in } D
$$

By (1.3) and (1.4), there exists a constant $\varepsilon_{2}>0$ such that

$$
\begin{gathered}
\left(\lambda_{1}-\lambda \frac{f\left(\varepsilon_{2} \varphi_{1}\right)}{\varepsilon_{2} \varphi_{1}}\right)>0 \text { in } D \\
\varepsilon_{2} \varphi_{1}<u_{\mu} \text { in } D .
\end{gathered}
$$

This implies that $\varepsilon_{2} \varphi_{1}$ is a strict super-solution of (1.1).

Summing up, we have constructed a strict sub-solution $u_{\mu}$, a strict super-solution $\varepsilon_{2} \varphi_{1}$, and a strict super-solution $\psi(\lambda)$ of (1.1). Furthermore, assertion (2.33) gives

$$
0<\varepsilon_{2} \varphi_{1}<u_{\mu}<\psi(\lambda) \text { in } D
$$

where $u \equiv 0$ is a sub-solution of (1.1). If we use Amann's three fixed point existence theorem [1, Theorem 14.2] to solve (1.1) in the framework of (2.19), then the strong increase of $F_{k}$ ensures the existence of at least two distinct nonnegative, nonzero solutions of (1.1) and then, they are positive in $D$ by the strong maximum principle.

The proof of Theorem 1.1 is now complete.

\section{Proof of Theorem 1.3}

This section is devoted to the estimate for the critical value $\Lambda$. We prove here that problem (1.1) has at least two distinct positive solutions in the open interval given by (1.17).

Our proof relies on the following lemma, which ensures the existence of at least three fixed points for equations of compact, nonnegative mappings in ordered Banach spaces (see [9, Lemma 4.4]).

Lemma 3.1. Let $X$ be an ordered Banach space with norm $\|\cdot\|$ and the positive cone $Q$ having nonempty interior, let $\eta: Q \rightarrow[0, \infty)$ be a continuous, concave functional and let $G$ be a compact mapping of $Q_{\tau}:=\{w \in Q:\|w\| \leq \tau\}$ into $Q$ for some constant $\tau>0$ such that

$$
\|G(w)\|<\tau, \quad \forall w \in \partial Q_{\tau} .
$$

Assume that there exist constants $0<\delta<\tau$ and $\sigma>0$ such that

$$
W=\left\{w \in \stackrel{\circ}{Q}_{\tau}: \eta(w)>\sigma\right\}
$$


204 Semilinear elliptic boundary value problems

is not empty, and that

$$
\begin{gathered}
\|G(w)\|<\delta, \quad \forall w \in \partial Q_{\delta}, \\
\eta(w)<\sigma, \quad \forall w \in Q_{\delta}, \\
\eta(G(w))>\sigma, \quad \forall w \in Q_{\tau} \text { satisfying } \eta(w)=\sigma .
\end{gathered}
$$

Then the mapping $G$ has at least three distinct fixed points in $Q_{\tau}$.

Let $\Omega$ be a sub-domain of $D$ with smooth boundary such that $\bar{\Omega} \subset D$. We put

$$
C_{\Omega}=\inf _{x \in \Omega} K \chi_{\Omega}
$$

where $\chi_{A}$ denotes the characteristic function of a subset $A$ of $D$, and put

$$
\beta=\sup _{\Omega} C_{\Omega}
$$

Here we note that $\beta$ is a positive constant because of the strict positivity of $K$.

Now we apply Lemma 3.1 to the case

$$
\begin{gathered}
X=C(\bar{D}), \\
Q=P=\{u \in C(\bar{D}): u(x) \geq 0 \text { in } \bar{D}\}, \\
G(\cdot)=F(\lambda, \cdot)=\lambda K f(\cdot), \\
\frac{1}{\bar{f} \beta}<\lambda<\frac{1}{\|e\|_{\infty}} .
\end{gathered}
$$

In this situation we verify (3.1), (3.3), (3.4), and (3.5). By the definitions of $\beta$ and $\bar{f}$ (see (1.10) and (3.7)), there exist a smooth sub-domain $\Omega$ of $D$ satisfying $\bar{\Omega} \subset D$, and a constant $t_{1}>0$ such that

$$
\lambda>\frac{t_{1}}{f\left(t_{1}\right) C_{\Omega}}
$$

for any $\lambda$ satisfying (1.17). Setting

$$
\eta(u)=\inf _{x \in \Omega} u(x)
$$

we find that $\eta$ is a nonnegative, continuous and concave functional on $P$. Since $f$ is nonnegative and nondecreasing, we have

$$
\inf _{x \in \Omega} \lambda K f(u) \geq \lambda \inf _{x \in \Omega} K\left(f(u) \chi_{\Omega}\right) \geq \lambda f\left(t_{1}\right) \inf _{x \in \Omega} K \chi_{\Omega}>t_{1}
$$

for any $u \in P$ satisfying that $\inf _{x \in \Omega} u(x)=t_{1}$. Hence condition (3.5) has been verified for $\sigma=t_{1}$.

Since $f_{\infty} \leq 1$, we obtain

$$
\lambda<\frac{1}{f_{\infty}\|e\|_{\infty}} .
$$


By (1.11), we can choose $t_{2} \in\left(t_{1}, \infty\right)$ large such that

$$
\lambda<\frac{t_{2}}{f\left(t_{2}\right)} \cdot \frac{1}{\|e\|_{\infty}} .
$$

This implies that if $u \in \partial P_{t_{2}}$, then we have

$$
\|\lambda K f(u)\|_{\infty}<t_{2}
$$

since $f$ is nondecreasing. Here we have used the fact that $e=K 1$. Hence condition (3.1) has been verified for $\tau=t_{2}$ and also, it is easily seen that the set

$$
W:=\left\{w \in \stackrel{\circ}{P}_{t_{2}}: \inf _{x \in \Omega} w>t_{1}\right\}
$$

is nonempty, since $t_{2}>t_{1}$.

From the condition

$$
\lambda<\frac{1}{\|e\|_{\infty}},
$$

conditions (1.3) and (1.4) ensure the existence of $t_{3} \in\left(0, t_{1}\right)$ small such that

$$
\lambda<\frac{t_{3}}{f\left(t_{3}\right)} \cdot \frac{1}{\|e\|_{\infty}} .
$$

In the same way as above, we have

$$
\|\lambda K f(u)\|_{\infty}<t_{3}
$$

for any $u \in \partial P_{t_{3}}$. Hence condition (3.3) has been verified for $\delta=t_{3}$.

Finally, we observe that if $u \in P_{t_{3}}$, then

$$
\inf _{x \in \Omega} u(x) \leq\|u\|_{\infty} \leq t_{3}<t_{1} .
$$

Hence condition (3.4) has been verified.

As a consequence of Lemma 3.1, we therefore conclude that (2.14) has at least three distinct fixed points in $P_{t_{2}}$. The same argument in Section 2 completes the proof of Theorem 1.3.

\section{Examples}

In this section, we give an example of nonlinearity $f$ satisfying the assumption of Theorem 1.3. Let $m$ be a positive constant and define $f_{m}$ of the form

$$
f_{m}(t)= \begin{cases}\tan t, & 0 \leq t \leq \arctan m, \\ m+(\arctan m)\left(1+m^{2}\right)\left(1-\frac{\arctan m}{t}\right), & t>\arctan m .\end{cases}
$$

Then we easily see that $f_{m}$ is continuously differentiable with respect to $t \geq 0$ and satisfies (1.3), (1.4), (1.8), and (1.9) with

$$
\left(f_{m}\right)_{\infty}=0
$$


206 Semilinear elliptic boundary value problems

It can be checked that $f_{m}$ is strictly increasing with respect to $t \geq 0$ and then, we have

$$
\bar{f}_{m}=\sup _{t>0} \frac{f_{m}(t)}{t} \geq \frac{f_{m}(\pi / 2)}{\pi / 2}>\frac{f_{m}(\arctan m)}{\pi / 2}=\frac{2 m}{\pi} .
$$

This implies that if

$$
m>\frac{\pi\|e\|_{\infty}}{2 \beta},
$$

then condition (1.16) holds for $f_{m}$.

Now we consider the solvability of the semilinear Neumann problem

$$
\begin{gathered}
(-\Delta+c) u=\lambda f_{m}(u) \quad \text { in } D, \\
\frac{\partial u}{\partial \mathbf{n}}=0 \quad \text { on } \partial D .
\end{gathered}
$$

Here $c$ is a positive constant and $f_{m}$ is given by (4.1).

To describe precisely the number of the positive solutions, the following lemma is proved, which gives an estimate for $\beta$ in the Neumann or Robin case.

Lemma 4.1. Assume

$$
0<a(x) \leq 1 \quad \text { on } \partial D
$$

Then we obtain

$$
\frac{1}{\|e\|_{\infty}} \leq \frac{1}{\beta} \leq \frac{1}{\min _{\bar{D}} e}
$$

Proof. In [10, Lemma 5.1] we can see that

$$
\frac{1}{\|e\|_{\infty}} \leq \frac{1}{\beta}
$$

It remains to show that

$$
\frac{1}{\beta} \leq \frac{1}{\min _{\bar{D}} e} .
$$

To do so, we choose a sequence $\left\{\Omega_{j}\right\}$ of relatively compact subdomains of $D$, with smooth boundary, such that $\Omega_{j} \uparrow D$ as $j \rightarrow \infty$. Let

$$
w_{\Omega_{j}}=K \chi_{\Omega_{j}} .
$$

Thanks to the Sobolev imbedding theorem, Theorem 2.2 gives

$$
\left\|w_{\Omega_{j}}-e\right\|_{\infty} \longrightarrow 0 \quad \text { as } j \longrightarrow \infty \text {. }
$$

In case (4.6), $w_{\Omega_{j}}$ and $e$ are both strictly positive on $\bar{D}$ and we obtain

$$
\begin{gathered}
\inf _{\Omega_{j}} w_{\Omega_{j}}=\sup _{\Omega_{j}} \frac{1}{w_{\Omega_{j}}}=\sup _{D} \frac{\chi_{\Omega_{j}}}{w_{\Omega_{j}}}, \\
\min _{\bar{D}} e=\inf _{D} e=\sup _{D} \frac{1}{e} .
\end{gathered}
$$


However, condition (4.11) gives

$$
\left\|\frac{\chi_{\Omega_{j}}}{w_{\Omega_{j}}}-\frac{1}{e}\right\|_{\infty} \longrightarrow 0 \text { as } j \longrightarrow \infty,
$$

so that

$$
\sup _{D} \frac{\chi_{\Omega_{j}}}{w_{\Omega_{j}}} \longrightarrow \sup _{D} \frac{1}{e} \text { as } j \longrightarrow \infty .
$$

In view of assertion (4.12), this implies

$$
\inf _{\Omega_{j}} w_{\Omega_{j}} \longrightarrow \min _{\bar{D}} e \quad \text { as } j \longrightarrow \infty .
$$

Therefore the desired inequality (4.9) follows from (4.15), since we have

$$
\inf _{\Omega_{j}} w_{\Omega_{j}} \leq \sup _{\bar{\Omega} \subset D} \inf _{\Omega} w_{\Omega}=\beta
$$

The proof of Lemma 4.1 is complete.

Now we have the following existence and multiplicity theorem for (4.5).

THEOREM 4.2. If $m>\pi / 2$, then there exists at least one positive solution of (4.5) for each

$$
\lambda \geq \frac{c}{\overline{f_{m}}}
$$

and no positive solution for any

$$
0<\lambda<\frac{c}{\bar{f}_{m}}
$$

Moreover, problem (4.5) has at least two positive solutions for every

$$
\frac{c}{\overline{f_{m}}}<\lambda<c .
$$

Proof. We first recall (1.19). Since $e=1 / c$ in this case, assertion (4.7) shows $1 / \beta=c$. In view of (4.4), Theorem 4.2 follows as a consequence of Theorems 1.1 and 1.3.

The proof of Theorem 4.2 is now complete.

\section{References}

[1] H. Amann, Fixed point equations and nonlinear eigenvalue problems in ordered Banach spaces, SIAM Rev. 18 (1976), no. 4, 620-709. MR 54\#3519. Zbl 345.47044.

[2] A. Ambrosetti, H. Brézis, and G. Cerami, Combined effects of concave and convex nonlinearities in some elliptic problems, J. Funct. Anal. 122 (1994), no. 2, 519-543. MR 95g:35059. Zbl 805.35028.

[3] P.-L. Lions, On the existence of positive solutions of semilinear elliptic equations, SIAM Rev. 24 (1982), no. 4, 441-467. MR 84a:35093. Zbl 511.35033. 
208 Semilinear elliptic boundary value problems

[4] K. Taira, Bifurcation for nonlinear elliptic boundary value problems. I, Collect. Math. 47 (1996), no. 3, 207-229. MR 98i:35067a. Zbl 865.35014.

[5] K. Taira and K. Umezu, Bifurcation for nonlinear elliptic boundary value problems. II, Tokyo J. Math. 19 (1996), no. 2, 387-396. MR 98i:35067b. Zbl 867.35009.

[6] Bifurcation for nonlinear elliptic boundary value problems. III, Adv. Differential Equations 1 (1996), no. 4, 709-727. MR 98i:35067c. Zbl 860.35039.

[7] Positive solutions of sublinear elliptic boundary value problems, Nonlinear Anal. 29 (1997), no. 7, 761-771. MR 98h:35082. Zbl 878.35048.

[8] Kenichiro Umezu, $L_{p}$-approach to mixed boundary value problems for second-order elliptic operators, Tokyo J. Math. 17 (1994), no. 1, 101-123. MR 95e:35057. Zbl 812.35033.

[9] H. Wiebers, S-shaped bifurcation curves of nonlinear elliptic boundary value problems, Math. Ann. 270 (1985), no. 4, 555-570. MR 86f:35027. Zbl 544.35015.

[10] Critical behaviour of nonlinear elliptic boundary value problems suggested by exothermic reactions, Proc. Roy. Soc. Edinburgh Sect. A 102 (1986), no. 1-2, 19-36. MR 87k:35095. Zbl 609.35073.

Kenichiro Umezu: Faculty of Liberal Arts and Sciences, Maebashi Institute of TechNOLOGY, MAEBASHI 371-0816, JAPAN

E-mail address: ken@maebashi-it.ac.jp 


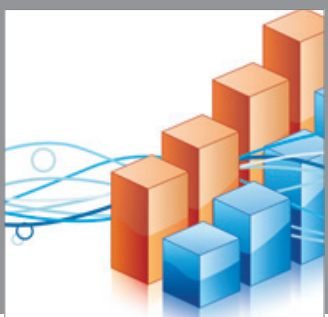

Advances in

Operations Research

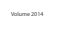

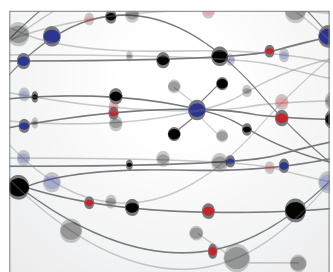

\section{The Scientific} World Journal
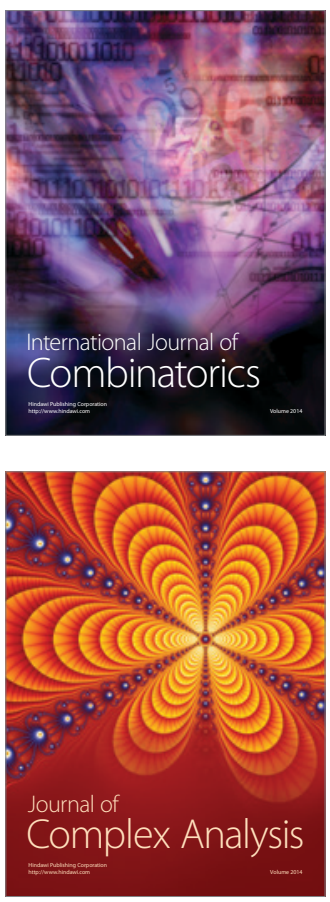

International Journal of

Mathematics and

Mathematical

Sciences
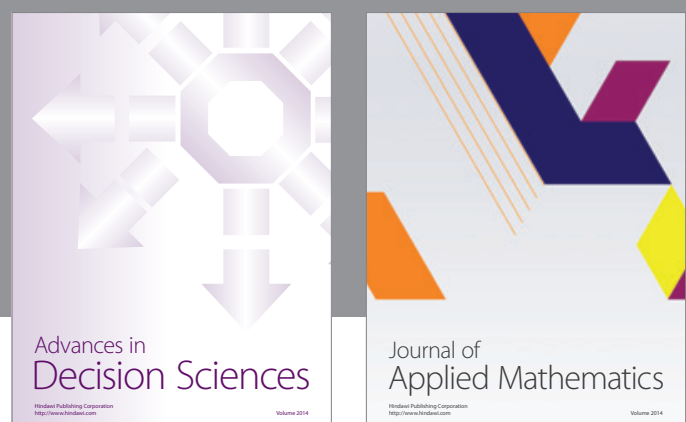

Journal of

Applied Mathematics
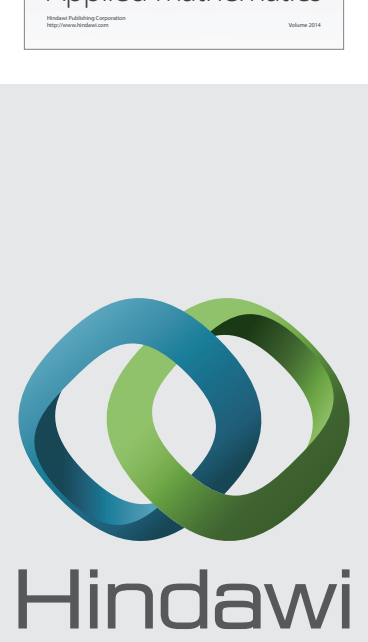

Submit your manuscripts at http://www.hindawi.com
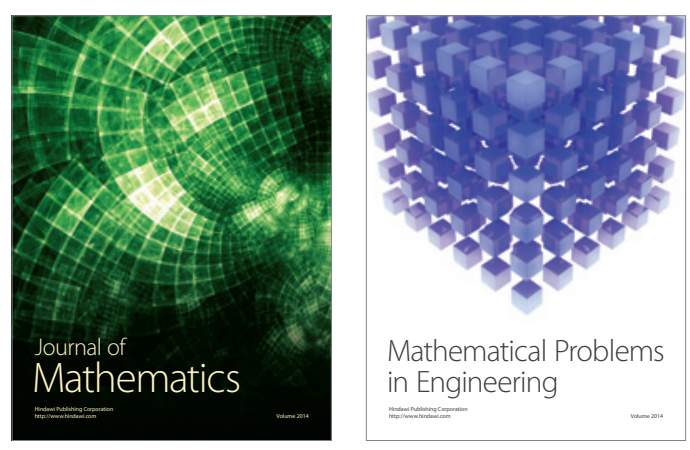

Mathematical Problems in Engineering
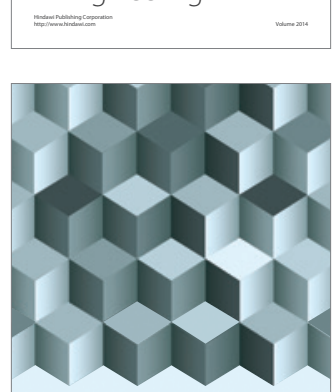

Journal of

Function Spaces
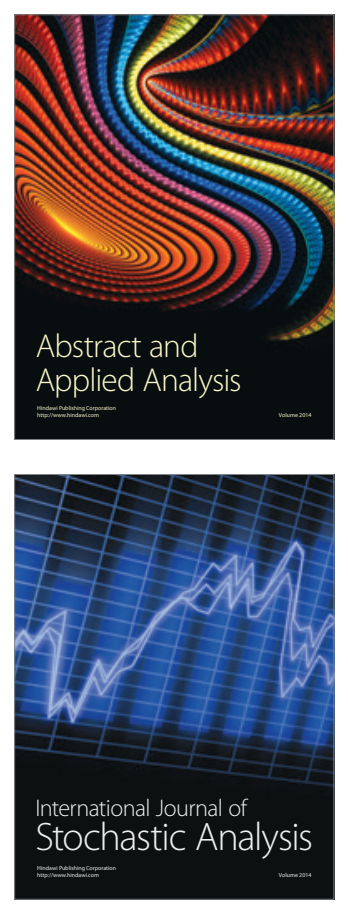

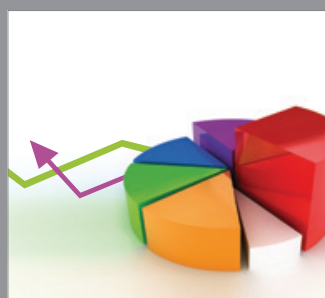

ournal of

Probability and Statistics

Promensencen
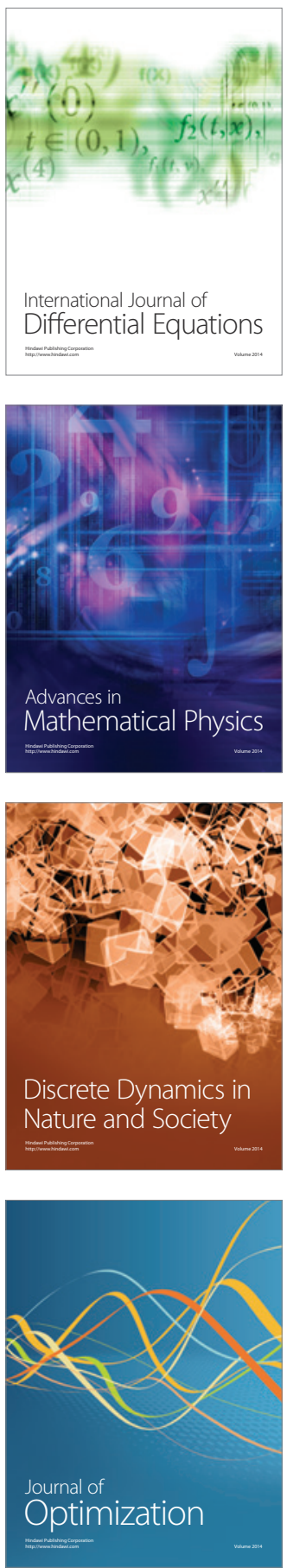